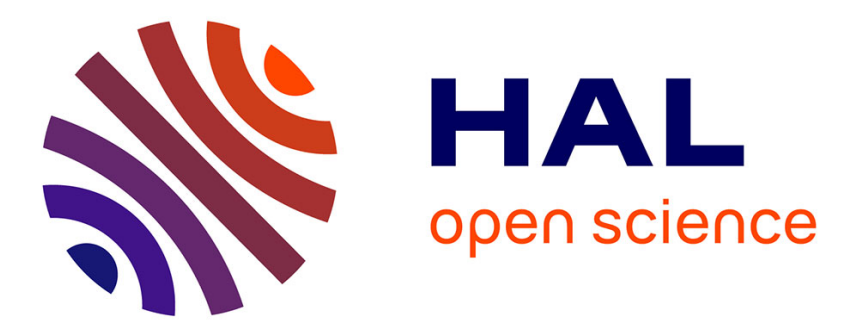

\title{
Estimation of Separating Planes between Touching 3D Objects Using Power Watershed
}

\author{
Clara Jaquet, Edward Andò, Gioacchino Cinno Viggiani, Hugues Talbot
}

\section{To cite this version:}

Clara Jaquet, Edward Andò, Gioacchino Cinno Viggiani, Hugues Talbot. Estimation of Separating Planes between Touching 3D Objects Using Power Watershed. 11th International Symposium on Mathematical Morphology and Its Applications to Signal and Image Processing (ISMM 2013), May 2013, Uppsala, Sweden. pp.452-463, 10.1007/978-3-642-38294-9_38 . hal-01617159

\section{HAL Id: hal-01617159 \\ https://hal.science/hal-01617159}

Submitted on 16 Oct 2017

HAL is a multi-disciplinary open access archive for the deposit and dissemination of scientific research documents, whether they are published or not. The documents may come from teaching and research institutions in France or abroad, or from public or private research centers.
L'archive ouverte pluridisciplinaire HAL, est destinée au dépôt et à la diffusion de documents scientifiques de niveau recherche, publiés ou non, émanant des établissements d'enseignement et de recherche français ou étrangers, des laboratoires publics ou privés. 


\title{
Estimation of separating planes between touching 3D objects using power watershed
}

\author{
Clara Jaquet ${ }^{1}$ and Edward Andò ${ }^{2}$ and Gioacchino Viggiani ${ }^{2}$ and Hugues Talbot ${ }^{1}$ \\ 1 Université Paris-Est, Laboratoire d'Informatique Gaspard-Monge, Equipe A3SI, ESIEE, \\ Noisy-le-Grand, France \\ 2 Grenoble-INP / UJF-Grenoble 1 / CNRS UMR 5521, Laboratoire 3SR, Grenoble, France.
}

\begin{abstract}
The problem of separating touching or overlapping objects is classical in imaging. Many solutions have been proposed in 2D. While similar, the problem in 3D has differentiating features. Contrary to the 2D case, apparent overlap due to projection effects does not exist, but real or apparent interpenetration can occur only due to either physical particle fusion or partial volume effects. Often the ability to separate objects logically is sufficient, however sometimes finding the orientation of tangent separating plane is useful. In this article, we propose a method for separating 3D touching objects and estimate a precise separating plane based on power watershed. Power watershed is used in two steps, first to obtain individual object identification, and in a second step to compute a probability field allowing sub-voxel accuracy in the plane fitting procedure. We show that our procedure is much more precise than a simple segmentation approach. We illustrate this in an application involving the shearing of a sample of sand grains imaged in various configurations by micro-CT tomography. Our technique allows the measurement of the orientation of the contacts between grains, a quantity that theoretical developments in soil mechanics explicitly use, but which has up until now been difficult to measure from experiments.
\end{abstract}

Keywords: Segmentation, random walker, orientations, micro-tomography.

\section{Introduction}

In this article, we aim to accurately measure the contact orientations between touching 3D particles.

Our motivation is to enable some experimental measurements in soil mechanics. When granular materials such as sand are mechanically loaded, strain localisation can manifest itself as shear banding which leads to rapid, non-linear failure of the material; failure is associated with catastrophic events such as landslides. In order to better understand the process of strain localisation in a granular material such as sand, it must be studied at the scale at which strain localises; for sands this is likely to be the grain scale. Historically micro-mechanical information has been difficult to measure in real experiments, so this information has been obtained by numerical simulations on large numbers of particles. Recent advances in X-ray tomography allow individual sand grains to be imaged in 3D, paving the way for grain-scale measurements on real granular materials. A full micro-mechanical description of the kinematics occurring at the grain scale needs 
to include grain kinematics (rotations and displacements of grains), as well as contact kinematics (the gain or loss of contacts, and the orientation of those contacts).

Fig 4 illustrates the process of strain localisation: vertical sections from 3D images of a sample show that a small subvolume of grains is massively deformed by the shear band that develops in the sample. In such a sample there are more than fifty thousand grains, so automatically and accurately segmenting each grain as well as providing unbiased estimates of grain contact orientation is essential for a complete micromechanical description of the processes at work.

In 2D images, separating binary overlapping convex objects is a common and classical problem in image analysis. It has been presented to students of Mathematical Morphology (MM) for a long time [3], and is typically solved with distance functions [17] and watershed [4]. It is one of the most well-known application of MM in general [10, 11]. This simple distance-based solution has many drawbacks. Proposed improvements over it have led to many fruitful concepts in MM, such as h-extrema [22], elliptical distance transforms [20] and conditional bisectors [14, 21,7].

In $3 \mathrm{D}$ the problem is similar but different. In $2 \mathrm{D}$ the need to separate overlapping objects is common due to projection effect, whereas in 3D this effect does not exist. However there exist some physical cases where interpenetrating (quasi) convex objects are grown or fused together such as sintering processes in materials science. As well, when objects are small, lack of resolution or diffusion in some imaging modalities may cause an effect known as partial volume effects to become prevalent [5]. This may causes 3D particles to appear fused rather than simply touching.

Separating fused objects in 3D is conceptually no different than in 2D, and indeed most methods developed for $2 \mathrm{D}$ readily carry over to higher dimensions with similar success. However the problem we study in this article is somewhat different again. In many applications, segmenting the fused objects is sufficient, allowing separated objects to be counted and measured. Nonetheless, when two objects are touching physically, it is sometimes useful to study the contact itself, in particular the spatial orientation of the plane of contact, when such a concept can be defined. We consider a correctly oriented separation plane between two grains to be a good approximation of a mechanically relevant contact orientation. A reliable and accurate way to automatically measure orientation of the contact surface between objects is therefore required.

The rest of the paper is organized as follows: in section 2 we provide the necessary background notions. In section 3 we describe our proposed segmentation and separating plane orientation estimation procedure. In section 4 we present results on simulated touching spherical particles. In section 5 we present results on real data, first on physical spherical beads, and then on actual sand grains. We conclude by showing that our procedure is less biased than previous methods for separating grains.

\section{Background notions}

Here we present the necessary notions for our proposed method. 


\subsection{Watersheds}

Watershed is the de-facto standard procedure for segmentation in MM [16]. Watershed as a segmentation procedure was proposed at the end of the 1970s [4], however fast procedures enabling its efficient computation were only proposed in the 1990s [23, 15]. Various types of watershed algorithms have been proposed. We may mention some that produce labeled regions with a thin inter-pixel boundaries like Meyer's algorithm [15], some that produce a thick pixel boundary like the topological watershed [8], and some that make explicit use of the graph structure of the underlying image like watershed cuts [9]. In the latter case, the resulting separating cut or boundary is located inter-pixel.

\subsection{Power watershed}

Recently a new type of watershed was introduced, the power watershed [6]. It is defined as the epi-convergence of the result of the optimization of the following energy:

$$
\underset{\mathbf{x}}{\operatorname{argmin}} \sum_{e_{i j} \in E} w_{i j}^{p}\left|x_{i}-x_{j}\right|^{q}+\sum_{v_{i} \in V} w_{i}^{p}\left|x_{i}-y_{i}\right|^{q} .
$$

where $(V, E)$ is a graph consisting of an edge set $E$ linked by vertices belonging to $V$. Vertex $i$ is denoted $v_{i}$; the edge linking $v_{i}$ to $v_{j}$ is denoted $e_{i j}$. The $w_{i j}$ are edge weighting that are given, and the $x_{i}$ are vertex value belonging to $\mathbf{x}$, the set of all the vertex values that we seek to optimize. In imaging, the pixel locations correspond to vertices and the edges represent the underlying neighborhood connectivity. In this paper we consider the nearest neighbour, i.e. the 6-connectivity for 3D. In a Maximum A Posteriori (MAP) framework, similarly to Graph Cuts, the first sum over edges is interpreted as a regularization or smoothness term, while the second sum over vertices is a data fidelity term. In particular, this data term can be easily used to enforce seeds for image segmentation, i.e. regions where the labelling $\mathbf{x}$ is known and fixed.

Finally, in (1), $p$ and $q$ are integer with specific interpretations. In particular, when $q$ is set to 2 , for every finite $p$, the solution to (1) represents the energy of a Random Walker (RW) [12]. If we make $p$ tend to infinity, the resulting $\mathbf{x}_{\infty}$ epi-converges to a unique solution, which is termed the power-watershed. Indeed, this $\mathbf{x}_{\infty}$ has many of the properties of a usual watershed. In particular if the data fidelity represents binary seeds, then $\mathbf{x}_{\infty}$ is a binary labelling everywhere equal to the result of a watershed cut, except on separating plateaus. These are the regions of constant edge weighting that are contested between the two labels. On these edge plateaus, the solution is that of a random walker.

In [6], an efficient algorithm is provided to compute this solution without having to resort to a convergence process. On contested plateaus, as in the RW result, the values of $\mathbf{x}_{\infty}$ can be interpreted as the probability of a discrete random walker moving from vertex to vertex along the edges of the graph to reach one label vs. the others. In the case with arbitrary $n$ markers, one needs to compute $n$ probability fields, however in the case of two markers $A$ and $B$, if the probability of reaching $A$ from some point is $p$, then the probability of reaching $B$ is $1-p$, therefore computing a single probability field is sufficient. These aspects will be essential in the next section. 


\subsection{Objects separation with classical and power watersheds}

The procedure for object separation with classical watershed is well known, but we describe it here for reference. We assume 3D volume images and a pair of interpenetrating, approximately convex objects.

1. Given a binary image $I$ containing the objects, an inner distance transform $E(I)$ is computed for all objects. In this article we used the Euclidean distance transform [18].

2. The maxima of this distance transform $\operatorname{MAX}(E(I))$ are used as seeds. To account for boundary noise, more robust h-maxima can be used instead [19].

3. The Euclidean distance map image is inverted, and a watershed transform is computed on it WS(-E(I)), stemming from the seeds computed at step 2.

This procedure finds a satisfactory separating surface, even if the objects are of different size, if the objects interpenetration is not too deep, which will be the case in our application. Depending on the watershed transform used, the output may be a labeled image, in which case the watershed surface is assumed to be the located between neighboring voxels with a different label, or an actual discrete surface composed of voxels.

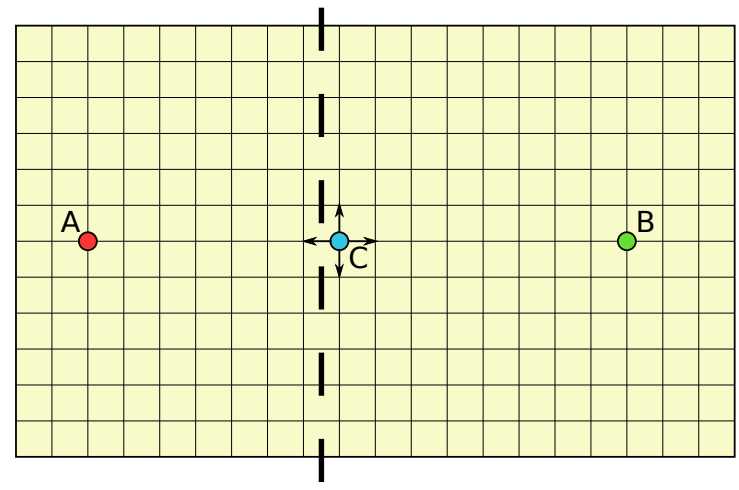

Fig. 1. Object separation with power watershed/random walker. Assuming a constant edge plateau, except on the thick vertical dotted line, where edges that are crossed are given a high weight, i.e. are difficult to cross. A and B represent labels. We estimate the probability of a random walker in $\mathrm{C}$ to move to the west as opposed to all the other directions (north, south or east). We see that locally the probabilities are identical because all neighboring edges at this point have the same weight. However globally, there are many ways for a random walker to come from $\mathrm{C}$ to $\mathrm{B}$. whereas there is only one way to go from $\mathrm{C}$ to A. Therefore, random walker probabilities must be high east of $\mathrm{C}$, and then drop rapidly through the vertical dotted line.

In the case of the power watershed, the procedure is different. Indeed, seeds are still needed, and can be extracted from the maxima of the Euclidean distance map, however the inverted distance map is not necessary for the computation of the labelling $\mathbf{x}$ described in section 2.2. Indeed, if we consider the binary object as a contested plateau, 
then the power watershed reduces to the computation of a pure random walker stemming from the disjoint seeds. In this case the computation of $\mathbf{x}$ on the plateau exhibits a sharp variation when crossing the boundary from one object domain to the next. This is due to the fact that a random walker is much more likely to reach the nearest seed in its domain of origin rather than cross the domain to the other seed through the small aperture between them. Therefore, to first order approximation, the resulting labelling $\mathbf{x}$ is almost binary on most of the contested plateau, with values very close to 1 near one seed, and close to 0 near the other. However a random walker placed close to the boundary between the two interpenetrating object is approximately as likely to reach either seed, and so the values of $\mathbf{x}$ in this area are close to 0.5 . To find a separating surface between the two objects, we can therefore consider the 0.5 -isosurface on $\mathbf{x}$. It has the advantage of a more precise location, benefiting from sub-pixel accuracy.

\section{Methodology}

This section details the method by which the surface that separates two touching objects in 3D can be accurately oriented. We describe our technique assuming a pair of touching objects. Due to unavoidable partial volume effects, these two objects appear slightly fused.

In this paper we present data from two sources: from simulations of Euclidean balls (section 4), and from 3D images of experiments of a granular material. The simulation directly produces a pair of contacting grains, whereas the images from experiments contain several thousands of grains; experimental data are therefore segmented and labelled using standard techniques in order to be able to extract pairs of grains.

Once a 3D image with two grains is obtained, one seed is identified inside each grain. In the simulations we simply use the centre of the balls. Seeds are defined in the experimental data segmenting grains from background. Seeds inside each grain are then computed using standard morphological operators: the 3D Euclidean distance map is calculated for the voxels describing the particles, and extended h-maxima of this map are identified. This procedure identifies two small volumes inside each grain.

The power watershed is then computed using these two seeds: the result of this is a map for each seeds describing the the probability of each voxel of belonging to one or the other of these seeds. As explained above, the 0.5 isosurface is identified. In fact is it sufficient for our application to the coordinates of a few points on this isosurface. For this, we associate every voxel in the pair of object with the label to which they most likely belong. This correspond to simply thresholding the probability map $\mathbf{x}_{\infty}$ at 0.5 . Then we identify voxels with neighbours that have a different label to their own, i.e. pairs of voxels that are on either side of the 0.5 isosurface. We then linearly interpolate along the line segment joining the center of each of these pairs of voxels in order to locate the 0.5 point with sub-pixel accuracy.

We record the list of these points and their coordinates and we finally fit a plane through these points using Principal Component Analysis (PCA). The normal to this plane is recorded as the orientation of the contact surface (or tangent) between the two grains. 
For comparison, we followed a similar procedure with several standard watersheds, namely the Meyer algorithm and the topological watershed. We also tried in one experiment a proprietary algorithm and watershed cuts. In cases were the algorithm yields a labeling with an inter-pixel separating surface, we took the midway point between pairs of pixels with different labels as being part of the separating surface. In cases where the algorithm yields a voxel-thick surface, we recorded the center of the voxels belonging to the surface. In all cases we performed a PCA analysis on the resulting point cloud to fit a plane and identified its normal as the orientation of the surface of contact between the two touching objects.

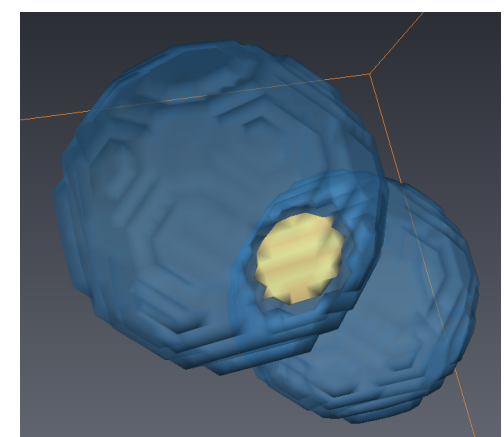

(a)

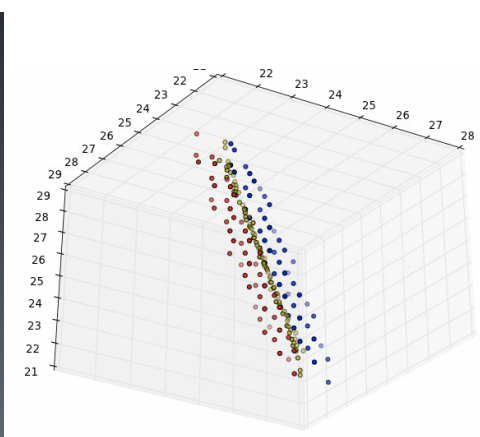

(b)

Fig. 2. Two touching balls. (a) shows the area of overlap in yellow and is close to a discrete disk. (b) shows the voxels on either side of the 0.5-isosurface in blue or red. In yellow the location of the interpixel 0.5 -isosurface. All points are very close to being located on the same plane.

\section{Testing on simulations}

In order to test our contact orientation measurement procedure, a simulation of particles with different orientations was performed. In this simulation two Euclidean balls of radius 10 pixels are defined in a 3D image and are placed in a random orientations so that their centres are 20 pixels apart (so that the balls are just touching). These balls are pixelised as binary objects.

Balls are used in this case since they have the unique property that the vector connecting their centres ("branch vector") has exactly the same orientation as the normal to the contact surface between the balls, which gives a ground truth to which measured orientations of the separation plane can be compared. Using the centres of the balls as seeds, the balls are then segmented using the technique outlined in the previous section. Fig. 2 shows the result of the segmentation and the computed location of some points on the 0.5 -isosurface defining the contact plane.

The computed orientation of the contact plane as an $(x, y, z)$ unit vector between the two balls can then be projected onto the unit $z$-positive half-sphere, since we cannot 
distinguish between either normals to the plane. To represent this projection, two numbers are sufficient, equivalent to a longitude and latitude. To represent these projections on 2D paper, we used the well-known Lambert polar equal-area projection [13], which is easy to compute. If orientations are identically and independently distributed on the unit half-sphere, this projection will yield a point distribution on a disk indistinguishable from a 2D Poisson point process.However, in the case of this simulation, because we can only readily simulate balls whose centers are located on a voxel, there is a finite number of possible orientations, and so our simulation shows a discrete bias.

Indeed, Fig 3 (a) shows the ground truth of evenly-distributed orientations obtained with a sufficient number of randomly places balls. Figure 3 (b) shows the orientations of the separation planes obtained with a standard topological watershed. Although the distribution of the orientations should be the same, it is clear that the topological watershed introduces some strong artefacts into the measured orientations, aligned at $45^{\circ}$ to the coordinate system. Figure 3 (c) shows the orientations of the separation planes obtained with the Meyer algorithm, in which the definition of the delimition between the two particles is interpixel. This appears to improve the isotropy of the distribution of orientations, which however remains very artefacted.

Figure 3 d) shows the distribution of orientations obtained with the measurement of the orientation of the separation place with the power watershed approach described above; it is obvious that the distribution of contact orientations obtained with this method is much closer to the true value. We now try our method on real data.

\section{Testing on real data}

The experimental data in this work come from tomographic images acquired on mechanical experiments on small samples of sand. Samples are prepared by "pluviating" the granular material into a cylindrical latex membrane measuring $22 \mathrm{~mm}$ height by 11 $\mathrm{mm}$ diameter. Sample are installed into a specifically-designed pressure cell; the cell is then filled with water and pressurised. The cell allows the sample to be rotated while it is imaged by x-rays - meaning that a 3D image of the sample can be obtained by reconstruction. The sample can then be deformed axially, by advancing a piston under displacement control (and measuring the reaction force), and re-imaged in-situ.

Previous work has shown that individual grain kinematics (displacements and rotations) can be obtained by tracking them with the methods developed in [2]. Figure 4 shows the macroscopic stress reponse of the sample, and at the bottom shows two vertical sections through the sample COEA01-GL (a sample of Caicos ooids, a very rounded natural sand) in two different configurations. On the left the sample is under isotropic compression, before axial deformation. On the right, the sample has undergone considerable deformation, has reached and passed the peak in axial strength and has developed a shear band. In these images, the sub-selection of grains under study are shown in black, and the rest of the grains in the sample are shown in grey. Particle tracking has bee used to follow these grains from COEA01-GL-01 to COEA01-GL-17.

In order to fully characterise the micro-mechanical evolution of the granular materials images, contact orientations are required, however the orientations measured are unusable due to strong artefacts introduced by the classical watershed used to segment 


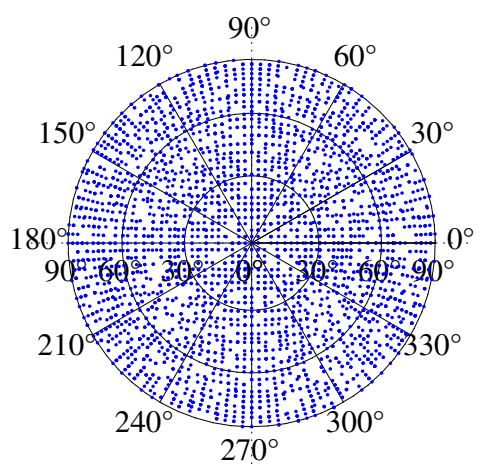

(a) Ground truth

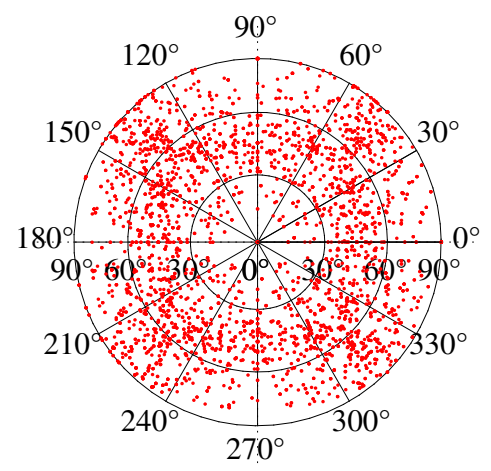

(c) Meyer algorithm

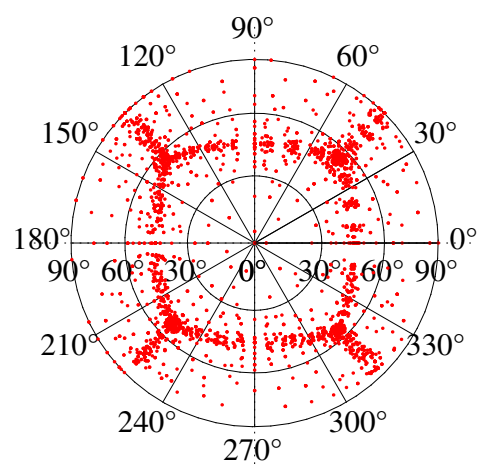

(b) Topological watershed

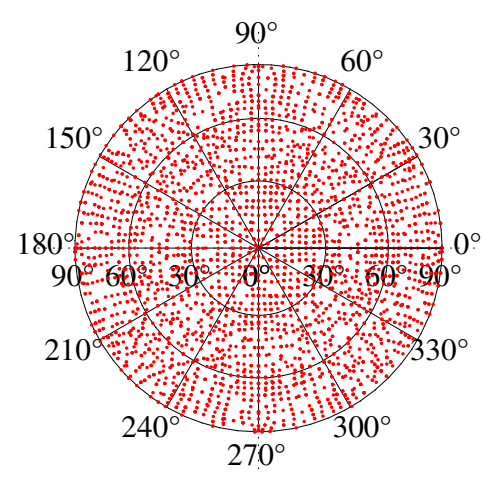

(d) Power watershed

Fig. 3. Equal area projection of orientations of separation planes from simulated balls.

particles [1]; these are probably aggravated by the fact that contact areas are relatively small in these images. A first test case will be performed on images coming from a sample (labelled ABEA02) of glass beads, which are close to perfect balls. This allows the same check to be performed as was done with the simulated balls: the branch vector can be calculated, giving a precise measurement of the expected contact orientation. Figure 5 a) shows the orientations of the branch vectors - which are distributed quite isotropically. The contact orientations coming from the power watershed shown in 5 (c) are much closer to the distribution of the branch vectors even in these real images - although around the $\pm x$ directions there appears to be some data missing. Overall this approach allows a new level of precision to be obtained. This means that contact orientations can be measured even without access to the branch vector.

Figure 6 shows the orientations that can be obtained with the technique presented in this paper, in the two configurations shown in figure 4. Immediately observable is 


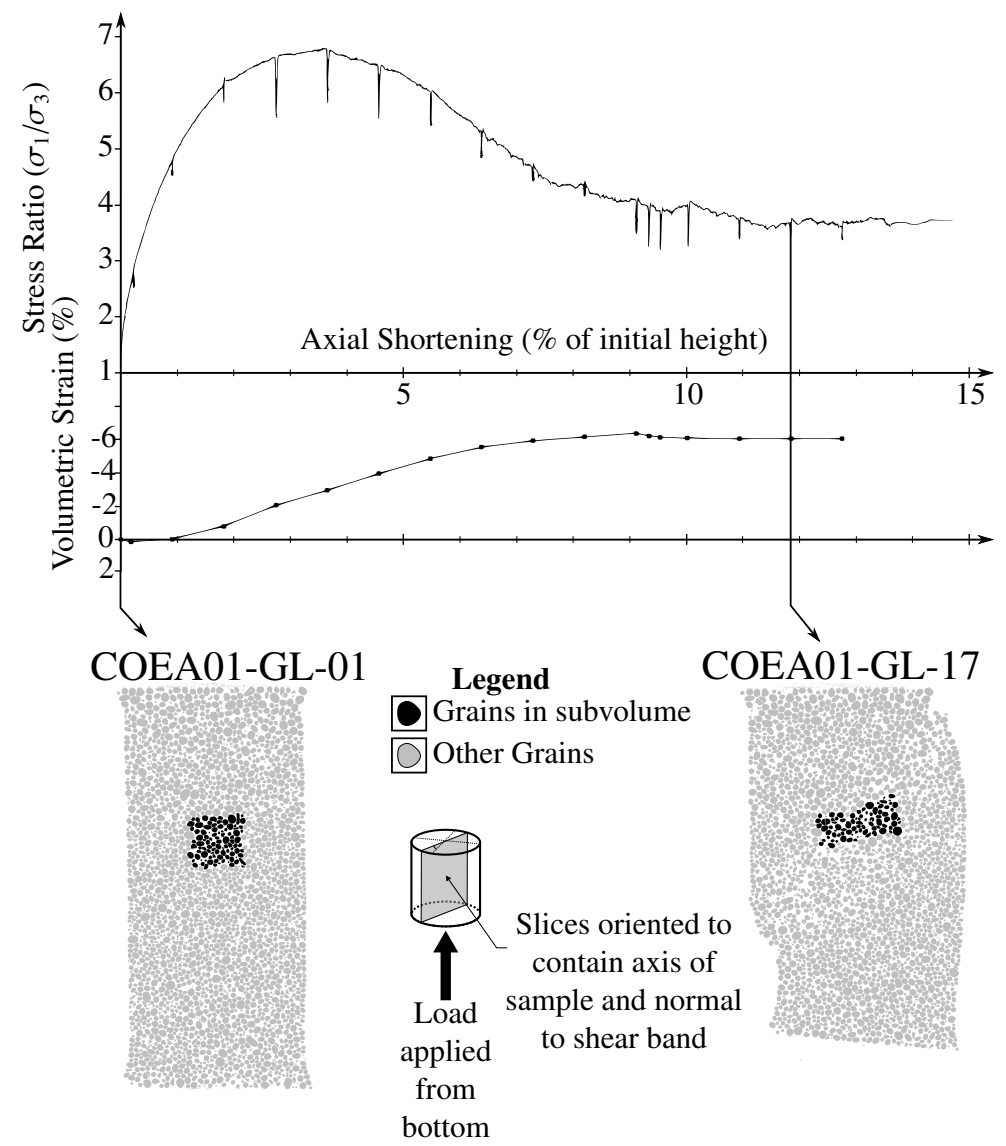

Fig. 4. Vertical cross sections from sample COEA01, oriented so as the contain the axis of the sample as well as the normal to the shear band which can be seen on the right. These slices have been taken along the $x$ direction, ( $z$ is the vertical axis)

the much more uniform distribution of contact orientations, when compared to standard techniques. Furthermore, a change in the distribution of orientations is visible between the two granular configurations.

In order to make this distribution easier to study, the projected orientations are divided into equal-area bins on the plane onto which they have been projected. The number of projected points that falls inside each bin is recorded, and bin totals are normalised by the median bin value. In figure 7 the colourmap is centred on 1 such that segments in white are those in which the median number of points has been counted, red ones show ones where more that the median has been counted, and blue shows where fewer than the median have been counted. This figure reveals that there is a small amount preferential alignment of the orientations towards the cardinal directions 


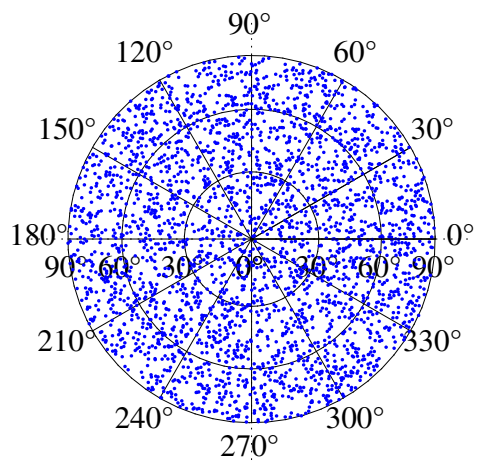

(a) Ground truth

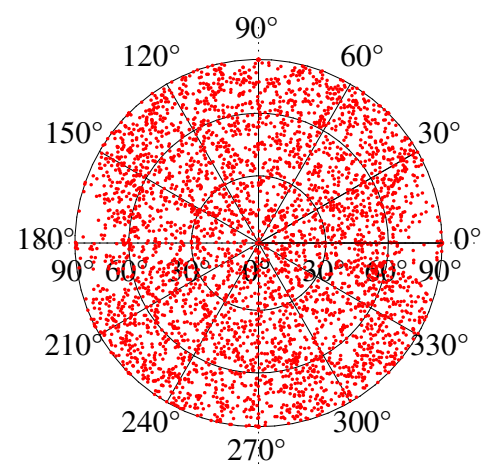

(b) Power watershed

Fig. 5. Equal area projection of orientations of contact between grains in sample ABEA02.

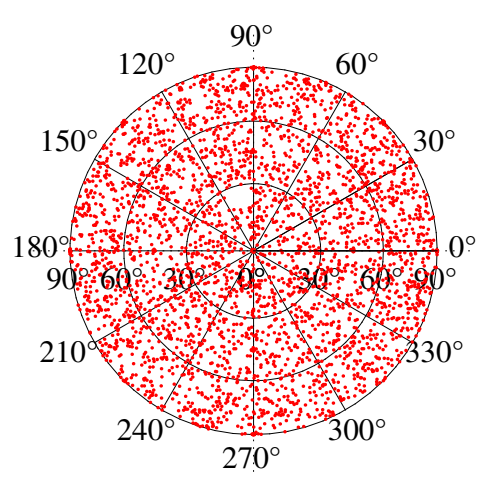

(a) COEA01-GL-01

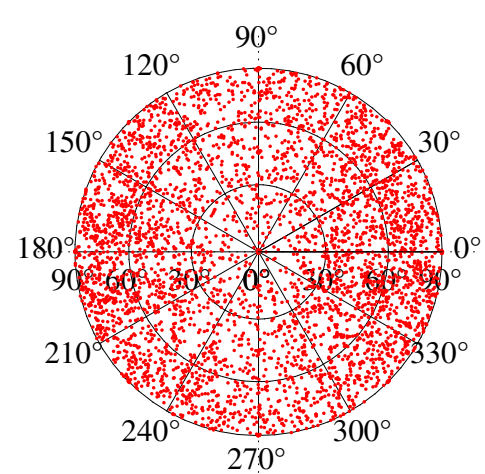

(b) COEA01-GL-17

Fig. 6. Orientations of contacts between grains in configurations COEA01-GL-01 and COEA01GL-17, as calculated using the power watershed.

(up, down, left, right, front, back), this is likely due to very small contact areas where the orientation can only be poorly defined.

From a mechanical point of view, a clear evolution in the distribution of contacts is visible: many contacts are gained in the $\mathrm{x}$-direction and many are lost in the $\mathrm{z}$-direction between configurations COEA01-GL-01 and COEA01-GL-17. Recalling that the subvolume of grains on which this is calculated becomes part of the shear band that forms (whose normal is approximately $[-1,-1,0]$ ), this seems to indicate that grain-to-grain contacts are lost in the direction of principal stress applied to the sample (along the axis of compression). Contacts are grained in the direction in which the shear band is advancing . 
COEA01-GL-01

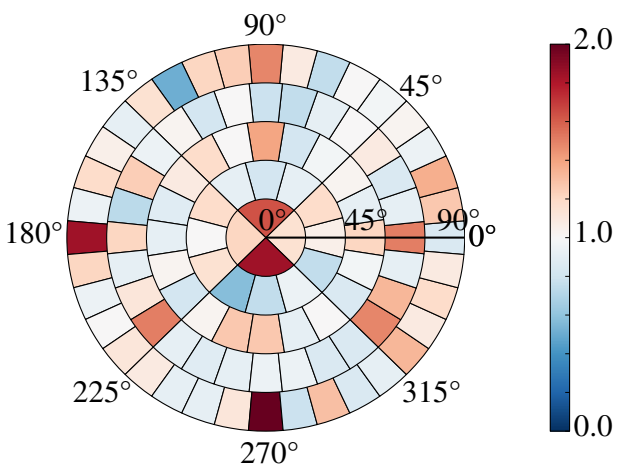

COEA01-GL-17

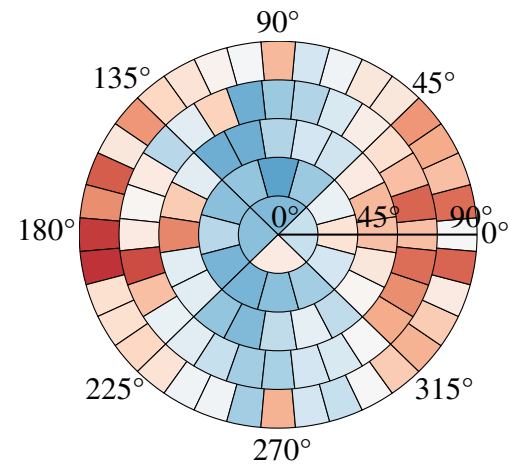

Fig. 7. Binned version of figure 6, where colours represent the number of projected points per bin divided by the mean number of projected points in all bins

\section{Discussion and conclusion}

In this article, we have proposed a new method based on power watershed (PW) to compute the orientation vector of the contact plane between touching objects in 3D micro-CT images. We rely on a number of assumptions, namely that the objects are nearly convex, that as a result the contact area is unique and somewhat regularized by partial volume effects. We consider touching objects in pairs using a method based on the standard watershed part of PW, and we compute the separating plane assuming the contact area is relatively small, using the random-walker part of PW. The contact plane is estimated with sub-voxel precision by estimating the equal-probability isosurface between the two objects. Due to the nature of the RW, we do not need to compute a distance map inside the two objects.

We have compared this approach to various standard watersheds combined with a Euclidean distance map. On simulated data, as well as physical bead and sand grains, we have show that our results are nearly free of artifacts and show a very good isotropic spread of measurements. Furthermore, we have been able to use this tool in a sand compression test, that shows conclusively that as pressure is applied, contact orientation anisotropy begins to develop in the sample.

This new technique paves the way towards a much more complete understanding of the micro-mechanics of real granular materials, enabling experimental data acquired on real granular media to be used in micro-mechanical models.

\section{References}

1. E. Andò, S. A. Hall, G. Viggiani, J. Desrues, and P. Bésuelle. Experimental micromechanics: grain-scale observation of sand deformation. Géotechnique Letters, 2(3):107-112, 2012b.

2. E. Andò, S.A. Hall, G. Viggiani, J. Desrues, and P. Bésuelle. Grain-scale experimental investigation of localised deformation in sand: a discrete particle tracking approach. Acta Geotechnica, pages 1-13, 2012. 
3. S. Beucher and C. Gratin. Micromorph reference manual, applications and solutions. Ecole des Mines de Paris, 1989.

4. S. Beucher and C. Lantuéjoul. Use of watersheds in contour detection. In Int. Workshop on Image Processing, Rennes, France, Sept. 1979. CCETT/IRISA.

5. I. Bloch. Some aspects of dempster-shafer evidence theory for classification of multimodality medical images taking partial volume effect into account. Pattern Recognition Letters, 17(8):905-919, 1996.

6. Camille Couprie, Leo Grady, Laurent Najman, and Hugues Talbot. Power watersheds: A unifying graph-based optimization framework. IEEE Transactions on Pattern Analysis and Machine Intelligence, 33(7):1384 - 1399, 2011.

7. Michel Couprie, David Coeurjolly, and Rita Zrour. Discrete bisector function and euclidean skeleton in 2d and 3d. Image Vision Computing, 25(10):1543-1556, 2007.

8. Michel Couprie, Laurent Najman, and Gilles Bertrand. Quasi-linear algorithms for the topological watershed. Journal of Mathematical Imaging and Vision, 22(2-3):231-249, May 2005. Special issue on Mathematical Morphology.

9. Jean Cousty, Gilles Bertrand, Laurent Najman, and Michel Couprie. Watershed cuts: thinnings, shortest-path forests and topological watersheds. IEEE Transactions on Pattern Analysis and Machine Intelligence, 32(5):925-939, May 2010.

10. John C.Russ. The image processing handbook, third edition. CRC Press, 1999.

11. R.C. Gonzalez and R.E. Woods. Digital Image Processing. Prentice-Hall, 3rd edition, 2007.

12. Leo Grady. Random walks for image segmentation. IEEE Transactions on Pattern Analysis and Machine Intelligence, 28(11):1768-1783, 2006.

13. A. R. Hinks. Map Projections, 2nd rev. ed. Cambridge University Press, Cambridge, England, 1921.

14. F. Meyer. Automatic screening of cytological specimens. Computer Vision, Graphics, and Image Processing, 35(3):356-369, 1986.

15. F. Meyer. Un algorithme optimal de ligne de partage des eaux. In Proc. 8ème Congrès Reconnaissance des Formes et Intelligence Artificielle, pages 847-857. AFCET, November 1991.

16. Fernand Meyer and Serge Beucher. Morphological segmentation. Journal on Visual Communication and Image Representation, 1(1):21-46, September 1990.

17. A. Rosenfeld and J.L. Pfaltz. Distance functions on digital pictures. Pattern Recognition, 1:33-61, 1968.

18. T. Saito and J.I. Toriwaki. New algorithms for euclidean distance transformation of an ndimensional digitized picture with applications. Pattern recognition, 27(11):1551-1565, 1994.

19. P. Soille. Morphological Image Analysis, principles and applications. Springer-Verlag, 2nd edition, 2003. ISBN 3-540-42988-3.

20. H. Talbot and B. Appleton. Elliptical distance transforms and the object splitting problem. In H. Talbot and R. Beare, editors, Mathematical Morphology, Proceedings of the 6th international symposium, pages 229-240, Sydney, Australia, April 2002. CSIRO Publishing.

21. H. Talbot and L. Vincent. Euclidean skeleton and conditional bisectors. In Visual Communications and Image Processing'92, volume 1818, pages 862-873, Boston, SPIE, November 1992.

22. L. Vincent. Morphological grayscale reconstruction in image analysis: Applications and efficient algorithms. Image Processing, IEEE Transactions on, 2(2):176-201, 1993.

23. L. Vincent and P. Soille. Watersheds in digital spaces: an efficient algorithm based on immersion simulations. IEEE Transactions on Pattern Analysis and Machine Intelligence, 13(6):583-598, June 1991. 